\title{
On Two Unicast Wireless Networks with Destination-to-Source Feedback
}

\author{
I-Hsiang Wang \\ EPFL, Lausanne, Switzerland \\ i-hsiang.wang@epfl.ch
}

\begin{abstract}
In this paper we study the role of feedback in layered two unicast wireless networks with arbitrary number of nodes and connectivity. The feedback model allows destinations to feedback their received signals to their respective sources. In the case of linear deterministic networks, we fully characterize the capacity region when the two individual minimum cut values are equal to 1 and show that feedback only helps increase capacity whenever the capacity region without feedback has $(1,1 / 2)$ or $(1 / 2,1)$ as its corner point but not both. Therefore, feedback helps balance the resource utilization of the two users, similar to the role of feedback in the two-user interference channel [1].
\end{abstract}

\section{INTRODUCTION}

In multi-user wireless networks, feedback helps balance the resource utilization among different users [1] by treating part of the interference received at destinations as useful side information. Through the feedback from destinations to sources, the side information can be exploited efficiently, and the system resource utilization is balanced across different users. In the context of the two-user interference channel, in the approximate characterization of the feedback capacity region, only the sum rate upper bound and the two individual rate bounds are active. In contrast, when feedback is not available [2], additionally bounds on $2 R_{1}+R_{2}$ and $R_{1}+2 R_{2}$ will be active, which implies that resource utilization is not balanced in certain regimes. The resource utilization interpretation [1] is best visualized using the linear deterministic model [3].

Channel output feedback in interference channels with constant channel gains has been well studied [4] [1] [5] [6]. With a good understanding on the role of feedback in single-hop multi-user networks, one of the natural follow-up questions is whether or not it extends to networks with arbitrary number of nodes and connectivity. In this paper we make a step towards answering this question by studying a class of layered two unicast linear deterministic networks [3] where the channel strengths are either unity or zero and the individual minimum cut values are equal to 1 . This class of networks was studied in the previous work [7] when feedback is not available. The non-feedback capacity region is completely characterized in [7], and similar to the two-user interference chanel [2], there are networks where in the characterization of the capacity region, bounds on $2 R_{1}+R_{2}$ or $R_{1}+2 R_{2}$ are active. Moreover, the class of two unicast linear deterministic networks can be partitioned into five different categories according to their capacity regions: $\left\{\mathfrak{T}, \mathfrak{T}_{12}, \mathfrak{T}_{21}, \mathfrak{P}, \mathfrak{S}\right\}$, as shown in Fig. 1 .

Our main result is that, when feedback is available from the destinations to their respective sources, it helps increase the capacity region if and only if the non-feedback capacity region of the network is $\mathfrak{T}_{12}$ or $\mathfrak{T}_{21}$. Moreover, the capacity region is always enlarged to the pentagon region $\mathfrak{P}$. Therefore, the category of networks with feedback capacity region being $\mathfrak{P}$ is the union of those with non-feedback capacity regions $\mathfrak{T}_{12}, \mathfrak{T}_{21}$, and $\mathfrak{P}$. In a word, the class of two unicast linear deterministic network can be classified into only three categories according to their feedback capacity regions: $\{\mathfrak{T}, \mathfrak{P}, \mathfrak{S}\}$. Since bounds on $2 R_{1}+R_{2}$ and $R_{1}+2 R_{2}$ are no longer active in characterizing the capacity region, in principle the role of feedback is similar to that in the two-user interference channel.

For the achievability, we provide a coding scheme that exploits feedback for utilizing side information at destinations for the category of networks with non-feedback capacity region $\mathfrak{T}_{12}$ (or $\mathfrak{T}_{21}$ ), so that the rate pair $(1,1 / 2)$ (or $(1 / 2,1)$ ) is achievable. For the outer bounds, we modify the proof of the non-feedback outer bounds by introducing new Markov relations taking the feedback into account, and show that the sum rate outer bounds still hold with the presence of feedback.

An important scenario of multiple unicast wireless networks is the Gaussian network. Recently, the degrees of freedom (DoF) region is completely characterized for layered two unicast Gaussian networks without feedback [8]. The result resembles that in the linear deterministic case [7] with a change of performance measure from rate to DoF. We conjecture that the conclusion of the current paper regarding the role of feedback can be extended to the Gaussian network. This direction is left as future work.

The rest of the paper is organized as follows. In Sec. II, we formulate the problem and define the network model. In Sec. III, we first review the non-feedback result adapted from [7] and provide the minimal necessary definitions for presenting the main theorem. Then in Sec. IV we present our feedback capacity result. A motivating example is given in Sec. V. The achievability proof is in Section VI, while the converse proofs are left in an extended version [9].

\section{Problem Formulation}

A two-source-two-destination layered network is a directed, acyclic, layered graph $\mathcal{G}=(\mathcal{V}, \mathcal{E})$, i.e. where the collection of nodes $\mathcal{V}$ can be partitioned into $L+2$ layers $(L \geq 0)$ :

$$
\mathcal{V}=\bigcup_{k=0}^{L+1} \mathcal{L}_{k}, \quad \mathcal{L}_{k} \cap \mathcal{L}_{j} \neq \emptyset, \forall k \neq j,
$$

such that for any edge $(\mathrm{u}, \mathrm{v}) \in \mathcal{E}, \exists k, 0 \leq k \leq L$ s.t. $\mathrm{u} \in$ $\mathcal{L}_{k}, \mathrm{v} \in \mathcal{L}_{k+1}$. The first layer $\mathcal{L}_{0}=\left\{\mathrm{s}_{1}, \mathrm{~s}_{2}\right\}$ consists of the 

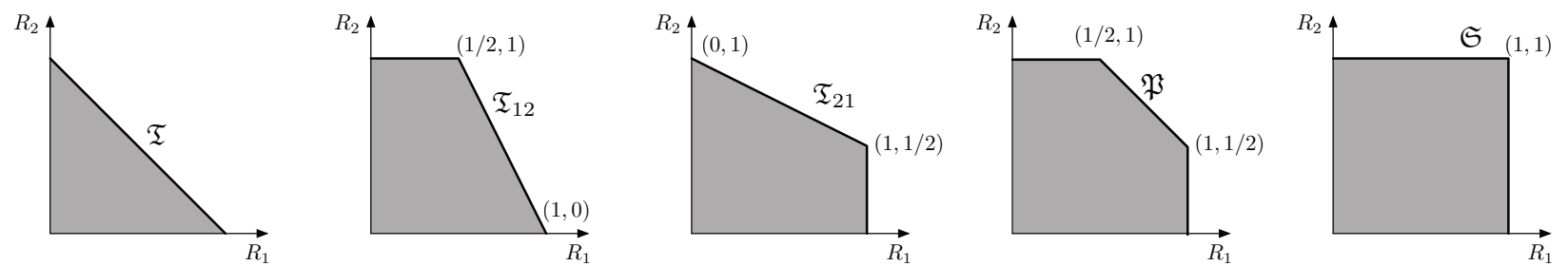

Fig. 1. Capacity Regions for Linear Deterministic Network. Without Feedback: $\left\{\mathfrak{T}, \mathfrak{T}_{12}, \mathfrak{T}_{21}, \mathfrak{P}, \mathfrak{S}\right\}$. With Feedback: $\{\mathfrak{T}, \mathfrak{P}, \mathfrak{S}\}$

two source nodes, and the last layer $\mathcal{L}_{L+1}=\left\{\mathrm{d}_{1}, \mathrm{~d}_{2}\right\}$ consists of the two destination nodes. Without loss of generality we assume each node in the network can be reached by at least one of the source nodes and can reach at least one of the destination nodes. For each node $v \in \mathcal{V} \backslash\left\{\mathrm{s}_{1}, \mathrm{~s}_{2}\right\}$, we define nodes that can reach $v$ as its predecessors. Let $\mathcal{P}(\mathrm{v})$ denote the set of predecessors that can reach $v$ in one step. We will call the nodes in $\mathcal{P}(\mathrm{v})$ as the parents of $\mathrm{v}$. A node is $\mathrm{s}_{i}$-reachable if it can be reached by $s_{i}$. It is $s_{i}$-only-reachable if it can be reached by $\mathrm{s}_{i}$ but not $\mathrm{s}_{j}, j \neq i$. It is $\mathrm{s}_{1} \mathrm{~s}_{2}$-reachable if it can be reached by both $s_{1}$ and $s_{2}$.

Each source $\mathrm{s}_{i}, i=1,2$, has its own message $W_{i}$ to be transmitted to its own destination $\mathrm{d}_{i}$ at rate $R_{i}$, and $\left\{W_{1}, W_{2}\right\}$ are independent. Let $X_{\mathrm{u}}[t]$ and $Y_{\mathrm{u}}[t]$ denote the transmitted and received signals of node $\mathrm{u}$ at time $t$ respectively. Due to the causal processing at each node, for any $t \geq 1$,

- $X_{\mathrm{s}_{i}}[t]$ is a function of $\left(W_{i}, Y_{\mathrm{d}_{i}}^{(t-1)}\right)$, for $i=1,2$

- $X_{\mathrm{u}}[t]$ is a function of $Y_{\mathrm{u}}^{(t-1)}$, for $\mathrm{u} \in \mathcal{V} \backslash\left\{\mathrm{s}_{1}, \mathrm{~s}_{2}\right\}$.

The notation $X^{t}:=\{X[1], \ldots, X[t]\}$ for $t \geq 1$ and $X^{0}:=\emptyset$.

Note that in the above formulation, we do not include the feedback links from the destinations to their respective sources into the graph $\mathcal{G}$. Hence, throughout this paper, any graph theoretic properties and conditions are associated to the graph $\mathcal{G}$ itself, not including the feedback links.

The channel model we consider is a special case of the linear deterministic network from [3]. The simplification is that if there is a link from one node to another, then the channel strength is unity. The reception of a node is the binary XOR of the transmission of its parents: $Y_{\mathrm{v}}[t]=\bigoplus_{\mathrm{u} \in \mathcal{P}(\mathrm{v})} X_{\mathrm{u}}[t]$.

\section{BACKGROUND [7]}

For completeness, below we first summarize the definitions in [7] for the case without feedback. These definitions play equally important roles in the feedback result. For a better exposition and illustraion please check [7]. Then we review the known result in the case without feedback, which lays the foundation for deriving the feedback result.

Definition 3.1 (Parents, Clones): For each $\vee \in \mathcal{V} \backslash\left\{\mathrm{s}_{1}, \mathrm{~s}_{2}\right\}$,

- let $\mathcal{P}(\mathrm{v})$ denote the set of parents of $\mathrm{v}$,

- let $\mathcal{P}^{s_{i}}(\mathrm{v}) \subseteq \mathcal{P}(\mathrm{v})$ denote the parents of $\mathrm{v}$ reachable by source $\mathrm{s}_{i}, i=1,2$,

- let $\mathcal{K}(\mathrm{v}):=\{\mathrm{u}: \mathcal{P}(\mathrm{u})=\mathcal{P}(\mathrm{v})\}$ denote the clones of $\mathrm{v}$, the set of nodes that receive the same signal as $v$,
- let $\mathcal{K}^{\mathrm{s}_{i}}(\mathrm{v}):=\left\{\mathrm{u}: \mathcal{P}^{\mathrm{s}_{i}}(\mathrm{u})=\mathcal{P}^{\mathrm{s}_{i}}(\mathrm{v})\right\}, i=1,2$, the set of nodes that have the same $\mathrm{s}_{i}$-reachable parents as $\mathrm{v}$. We called these nodes the $\mathrm{s}_{i}$-clones of $\mathrm{v}$.

Definition 3.2 (Vertex Cut): For two sets of nodes $\mathcal{U}_{1}$ and $\mathcal{U}_{2}$, we say a collection of nodes $\mathcal{T}$ is a $\left(\mathcal{U}_{1} ; \mathcal{U}_{2}\right)$-vertex-cut if in the graph obtained from the deletion of $\mathcal{T}$, there are no paths from any node in $\mathcal{U}_{1} \backslash \mathcal{T}$ to any node in $\mathcal{U}_{2} \backslash \mathcal{T}$. Note that this definition allows $\mathcal{T}$ to have nodes from $\mathcal{U}_{1}$ or $\mathcal{U}_{2}$.

Definition 3.3 (Omniscient Nodes): We say a node $\vee \in \mathcal{V}$ is omniscient if it satisfies either of (A) or (B) below:

(A) $\mathcal{K}(\mathrm{v})$ is a $\left(\mathrm{s}_{1}, \mathrm{~s}_{2} ; \mathrm{d}_{1}\right)$-vertex-cut and $\mathcal{K}^{\mathrm{s}_{2}}(\mathrm{v})$ is a $\left(\mathrm{s}_{2} ; \mathrm{d}_{2}\right)$ vertex-cut.

(B) $\mathcal{K}(\mathrm{v})$ is a $\left(\mathrm{s}_{1}, \mathrm{~s}_{2} ; \mathrm{d}_{2}\right)$-vertex-cut and $\mathcal{K}^{\mathrm{s}_{1}}(\mathrm{v})$ is a $\left(\mathrm{s}_{1} ; \mathrm{d}_{1}\right)$ vertex-cut.

Definition 3.4 (Critical Nodes): For each $i=1,2$, we define the critical node $v_{i}^{*}$ as any node with the smallest possible layer index such that $\mathcal{K}\left(\mathrm{v}_{i}^{*}\right)$ is a $\left(\mathrm{s}_{1}, \mathrm{~s}_{2} ; \mathrm{d}_{i}\right)$-vertex-cut. We use $\mathcal{L}_{k_{i}^{*}}$ to denote the layer where critical nodes $v_{i}^{*}$ lies.

A key property of the critical node $v_{i}^{*}$ is that, its reception completely determines the reception of destination $\mathrm{d}_{i}$.

Definition 3.5 (Cut Value and Min-Cut): Fix a set of nodes in layer $k, \mathcal{U} \subseteq \mathcal{L}_{k}$. Consider a partition of $\mathcal{V}$ into $\left(\mathcal{T}, \mathcal{T}^{c}\right)$ with $\mathrm{s}_{1}, \mathrm{~s}_{2} \in \mathcal{T}$ and $\mathcal{U} \subseteq \mathcal{T}^{c}$. Construct the transfer matrix $G$ with rows indexed by elements of $\mathcal{T}$ and columns indexed by elements of $\mathcal{T}^{c}$ where the $(\mathrm{u}, \mathrm{w})$ entry of $G$ is 1 if there is a directed edge from $u$ to $w$ and 0 otherwise. The rankmincut [3] from $\left\{\mathrm{s}_{1}, \mathrm{~s}_{2}\right\}$ to $\mathcal{U}$ is defined as the minimum rank of the transfer matrix $G$ over all such partitions $\left(\mathcal{T}, \mathcal{T}^{c}\right)$, and is denoted by $\mathrm{C}\left(\mathrm{s}_{1} \cdot \mathrm{s}_{2} ; \mathcal{U}\right)$.

Once we define the cut value, we can define primary mincut nodes for any set of nodes $\mathcal{U}$ with $\mathrm{C}\left(\mathrm{s}_{1}, \mathrm{~s}_{2} ; \mathcal{U}\right)=1$, due to the following lemma. What these primary min-cut nodes receive determines what $\mathcal{U}$ receive.

Lemma 3.1 (Primary Min-Cut): By $\mathcal{U}_{l}, 0 \leq l<k$, denote the set of nodes in layer $\mathcal{L}_{l}$ that can reach some node in $\mathcal{U}$. Let $l^{*}$ be the minimum index such that $\mathrm{C}\left(\mathrm{s}_{1}, \mathrm{~s}_{2} ; \mathcal{U}_{l^{*}}\right)=1$. Then, $\mathcal{U}_{l^{*}} \subseteq \mathcal{K}(\mathrm{u})$ for any $\mathrm{u} \in \mathcal{U}_{l^{*}}$, i.e. nodes in $\mathcal{U}_{l^{*}}$ are all clones of each other. We then define any of the nodes in $\mathcal{K}(\mathrm{u})$ as the primary min-cut node of $\mathcal{U}$, denoted by $\operatorname{Pmc}(\mathcal{U})$. It is unique up to clones.

Note that the reception of any node in $\mathcal{U}$ is a function of the reception of $\operatorname{Pmc}(\mathcal{U})$.

Next, we define induced graph $\mathcal{G}_{12}(\mathrm{w})$ for a node $w \in$ $\mathcal{P}^{\mathrm{s}_{2}}\left(\mathrm{v}_{1}^{*}\right)$ as follows. The purpose of these induced graphs is two-fold: 1) to capture the effect on the rest of the network 
caused by interference neutralization for $(1,1)$-achievability, and 2) to capture the Markov relations that are useful in the derivation of outer bounds.

Definition 3.6 (Induced Graph $\mathcal{G}_{12}$ ): If the min-cut value $\mathrm{C}\left(\mathrm{s}_{1}, \mathrm{~s}_{2} ; \mathcal{P}^{\mathrm{s}_{2}}\left(\mathrm{v}_{1}^{*}\right)\right)=2$ then define $\mathcal{G}_{12}(\mathrm{w}):=\mathcal{G}$. If the mincut value $\mathrm{C}\left(\mathrm{s}_{1}, \mathrm{~s}_{2} ; \mathcal{P}^{\mathrm{s}_{2}}\left(\mathrm{v}_{1}^{*}\right)\right)=1$, then we define $\mathcal{G}_{12}(\mathrm{w})$ as the graph obtained by modifying only the parents of nodes in $\mathcal{L}_{k_{1}^{*}}$ as follows. For $\mathrm{u} \in \mathcal{L}_{k_{1}^{*}}$,

$$
\mathcal{P}_{\mathcal{G}_{12}(\mathrm{w})}(\mathrm{u})= \begin{cases}\mathcal{P}(\mathrm{u}) & \text { if } \mathrm{w} \notin \mathcal{P}(\mathrm{u}) \\ \mathcal{P}(\mathrm{u}) \Delta \mathcal{P}^{\mathrm{s}_{2}}\left(\mathrm{v}_{1}^{*}\right) & \text { if } \mathrm{w} \in \mathcal{P}(\mathrm{u}),\end{cases}
$$

where $\Delta$ denotes symmetric set difference: $A \Delta B:=(A \backslash$ $B) \cup(B \backslash A)$. We then drop nodes in $\mathcal{G}_{12}(\mathrm{w})$ that cannot be reached by either of the two sources. In the rest of this paper, a graph theoretic object with a graph (say, $\mathcal{G}_{12}$ ) in its subscript, like $\mathcal{P}_{\mathcal{G}_{12}(\mathrm{w})}(\mathrm{u})$ above, denote the graph theoretic object in the induced graph $\mathcal{G}_{12}$.

Similarly we can define $\mathcal{G}_{21}(\mathrm{w})$ with indices 1 and 2 swapped.

We will use $\mathcal{G}_{12}(\mathrm{w})$ when $k_{1}^{*} \leq k_{2}^{*}$ and $\mathcal{G}_{21}(\mathrm{w})$ when $k_{2}^{*} \leq$ $k_{1}^{*}$. We will only use these graphs in relation to whether or not there is an omniscient node in $\mathcal{G}_{12}(\mathrm{w})$. Lemma 3.2 below allows us to drop the w and refer to any of the $\mathcal{G}_{12}(\mathrm{w})$ as $\mathcal{G}_{12}$ and talk about whether there is an omniscient node in $\mathcal{G}_{12}$.

Lemma 3.2: Suppose, in a network with no omniscient node, and with $k_{1}^{*} \leq k_{2}^{*}$, there exists a node $\mathrm{w}_{0} \in \mathcal{P}^{\mathrm{s}_{2}}\left(\mathrm{v}_{1}^{*}\right)$ such that there is an omniscient node in $\mathcal{G}_{12}\left(\mathrm{w}_{0}\right)$. Then for any node $\mathrm{w} \in \mathcal{P}^{\mathrm{s}_{2}}\left(\mathrm{v}_{1}^{*}\right)$, there is an omniscient node in $\mathcal{G}_{12}(\mathrm{w})$.

We define $\mathrm{T}^{(12)}$ as the conjunction of the following:

- $\mathrm{T}_{1}^{(12)}: 0<k_{1}^{*} \leq k_{2}^{*}$.

- $\mathrm{T}_{2}^{(12)}: \mathrm{C}\left(\mathrm{s}_{1}, \mathrm{~s}_{2} ; \mathcal{P}^{\mathrm{s}_{2}}\left(\mathrm{v}_{1}^{*}\right)\right)=1 . \mathrm{w}_{12}:=\operatorname{Pmc}\left(\mathcal{P}^{\mathrm{s}_{2}}\left(\mathrm{v}_{1}^{*}\right)\right)$.

- $\mathrm{T}_{3}^{(12)}$ : Let $\mathrm{u}_{21}:=\operatorname{Pmc}_{\mathcal{G}_{12}}\left(\mathrm{v}_{2}^{*}\right)$. $\mathrm{u}_{21}$ is omniscient in $\mathcal{G}_{12}$.

- $\mathrm{T}_{4}^{(12)}: \mathrm{w}_{12}=\mathrm{s}_{2}$, i.e., $\mathcal{P}^{\mathrm{s}_{2}}\left(\mathrm{v}_{1}^{*}\right)$ cannot be reached by $\mathrm{s}_{1}$.

Symmetrically, we can define condition $\mathrm{T}^{(21)}$ simply by swapping the indices 1 and 2 in the superscript.

Similarly, define $\mathrm{P}^{(12)}$ as the conjunction of the following:

- $\mathrm{P}_{1}^{(12)} \equiv \mathrm{T}_{1}^{(12)}, \mathrm{P}_{2}^{(12)} \equiv \mathrm{T}_{2}^{(12)}, \mathrm{P}_{3}^{(12)} \equiv \mathrm{T}_{3}^{(12)}$

- $\mathrm{P}_{4}^{(12)}: \mathrm{w}_{12} \neq \mathrm{s}_{2}$ and $\mathcal{K}^{\mathrm{s}_{2}}\left(\mathrm{w}_{12}\right)$ forms an $\left(\mathrm{s}_{2} ; \mathrm{d}_{2}\right)$-vertexcut in $\mathcal{G}$.

Finally, define $\mathrm{Q}^{(12)}:=\mathrm{T}^{(12)} \vee \mathrm{P}^{(12)}$ and it is the conjunction of the following:

- $\mathrm{Q}_{1}^{(12)} \equiv \mathrm{T}_{1}^{(12)}, \mathrm{Q}_{2}^{(12)} \equiv \mathrm{T}_{2}^{(12)}, \mathrm{Q}_{3}^{(12)} \equiv \mathrm{T}_{3}^{(12)}$

- $\mathrm{Q}_{4}^{(12)}: \mathcal{K}^{\mathrm{s}_{2}}\left(\mathrm{w}_{12}\right)$ forms an $\left(\mathrm{s}_{2} ; \mathrm{d}_{2}\right)$-vertex-cut in $\mathcal{G}$.

We use the following shorthand notations: $\mathrm{O}:=$ $\{\exists$ an omniscient node $\}, T:=T^{(12)} \vee T^{(21)}, P:=P^{(12)} \vee P^{(21)}$, and $\mathrm{Q}:=\mathrm{Q}^{(12)} \vee \mathrm{Q}^{(21)}=\mathrm{T} \vee \mathrm{P}$. Also, in the context that no confusion will be caused, we use the same notation to denote the set of networks that satisfy the condition.

Theorem 3.1 (Capacity Region without Feedback [7]):

The capacity region of two unicast flows over the linear deterministic networks without feedback is characterized as follows: the left-hand side denotes the collection of networks satisfying the condition, and the right-hand side denotes the capacity region of that collection of networks.

$\mathrm{O}$

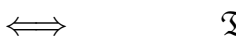

$\begin{array}{lll}\mathrm{T}^{(12)} \backslash \mathrm{O} & \Longleftrightarrow & \mathfrak{T}_{12} \\ \mathrm{~T}^{(21)} \backslash \mathrm{O} & \Longleftrightarrow & \mathfrak{T}_{21} \\ \mathrm{P} \backslash(\mathrm{T} \cup \mathrm{O}) & \Longleftrightarrow & \mathfrak{P} \\ \overline{\mathrm{Q}} \backslash \mathrm{O} & \Longleftrightarrow & \mathfrak{S}\end{array}$

\section{MAin Result}

The following theorem summarizes the main result for the two unicast linear deterministic networks with feedback.

Theorem 4.1 (Capacity Region with Feedback): The capacity region of two unicast flows over the linear deterministic networks with destination-to-source feedback is characterized as follows:

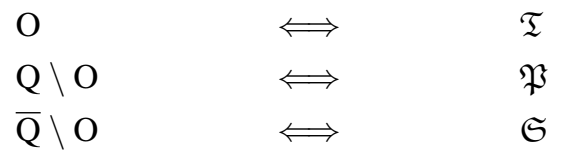

Remark 4.1: Note that compared to the case without feedback as shown in Theorem 3.1, the only difference is that, in any network belonging to the class $\mathrm{T}^{(12)} \backslash \mathrm{O}$ or the class $\mathrm{T}^{(21)} \backslash \mathrm{O}$, one can make use of feedback to enlarge the capacity region to the pentagon region $\mathfrak{P}$. Therefore, to prove Theorem 4.1, we only need to prove the following:

1) Achievability: By symmetry, it suffices to show that for a network that belongs to $\mathrm{T}^{(12)} \backslash \mathrm{O}$, when destination-to-source feedback is available, the rate pair $(1,1 / 2)$ is achievable.

2) Converse: Show that for a network that belongs to the class $\mathrm{O}$, its achievable sum rate $R_{1}+R_{2} \leq 1$, and for a network that belongs to the class $\mathrm{Q} \backslash \mathrm{O}, 2 R_{1}+2 R_{2} \leq 3$.

Due to space constraint, proof of the converse part is left in [9]. Below we summarize the two key lemmas that prove the converse part, and the details can be found in [9].

Lemma 4.1: If there exists an omniscient node as defined in Definition 3.3 in the two-unicast linear deterministic network, then for any achievable rate pair $\left(R_{1}, R_{2}\right), R_{1}+R_{2} \leq 1$.

Lemma 4.2: If the two-unicast linear deterministic network satisfies condition $\mathrm{Q}$, then for any achievable rate pair $\left(R_{1}, R_{2}\right), 2 R_{1}+2 R_{2} \leq 3$.

\section{A Motivating Example}

Before we go into the details of the proof, let us investigate an example network that belongs to the class $\mathrm{T}^{(12)} \backslash \mathrm{O}$, and show that indeed $\left(R_{1}, R_{2}\right)=(1,1 / 2)$ is achievable if sourceto-destination feedback is available. Moreover the sum rate $R_{1}+R_{2}$ is still upper bounded by $3 / 2$.

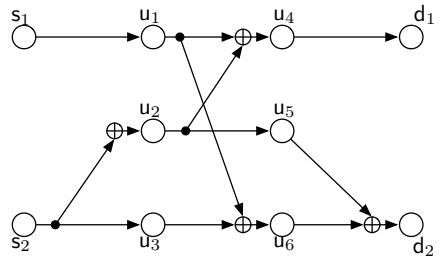

(a) Original Network

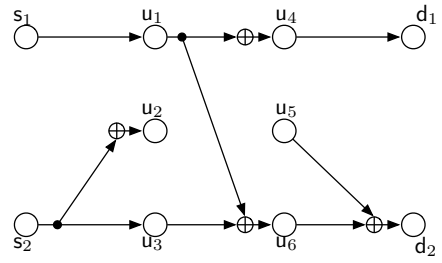

(b) Induced Graph $\mathcal{G}_{12}$
Fig. 2. An Example Network 
Consider the example illustrated in Fig. 2(a). First of all, let us check its properties and show that it satisfies the condition $\mathrm{T}^{(12)}$. In this example, the critical node for user 1 is the node $\mathrm{u}_{4}$, while that for user 2 is the destination $\mathrm{d}_{2}$. Moreover, it is easy to check that there is no omniscient node by checking the two critical nodes [7]. In particular, for the node $u_{4}$, its $\mathrm{s}_{2}$-reachable parents $\mathcal{P}^{\mathrm{s}_{2}}\left(\mathrm{u}_{4}\right)=\left\{\mathrm{u}_{2}\right\}$ and hence its $\mathrm{s}_{2}$ clones $\mathcal{K}^{\mathrm{s}_{2}}\left(\mathrm{u}_{4}\right)=\left\{\mathrm{u}_{4}, \mathrm{u}_{5}\right\}$. But the removal of $\left\{\mathrm{u}_{4}, \mathrm{u}_{5}\right\}$ cannot separate $d_{2}$ from $s_{2}$, and hence the node $u_{4}$ does not satisfy the definition of an omniscient node.

Next, let us check that condition $\mathrm{T}^{(12)}$ is satisfied.

- $\mathrm{T}_{1}^{(12)}$ : Here the critical node for user 1 lies in a layer with index smaller than that for user 2. Hence $k_{1}^{*}<k_{2}^{*}$.

- $\mathrm{T}_{2}^{(12)}$ : The critical node for user $1, \mathrm{u}_{4}$, has only one $\mathrm{s}_{2}$ reachable parent, which is the node $\mathrm{u}_{2}$. Hence we have $\mathrm{C}\left(\mathrm{s}_{1}, \mathrm{~s}_{2} ; \mathcal{P}^{\mathrm{s}_{2}}\left(\mathrm{u}_{4}\right)\right)=1$. Moreover, since $\mathrm{u}_{2}$ can only be reached by $\mathrm{s}_{2}$, the primary min-cut node of it, $\mathrm{w}_{12}=$ $\operatorname{Pmc}\left(\mathrm{u}_{2}\right)=\mathrm{s}_{2}$.

- $\mathrm{T}_{3}^{(12)}$ : The induced graph $\mathcal{G}_{12}\left(\mathrm{u}_{2}\right)$ is depicted in Fig. 2(b). Hence we see that indeed $\mathrm{u}_{21}:=\operatorname{Pmc}_{\mathcal{G}_{12}}\left(\mathrm{~d}_{2}\right)=\mathrm{u}_{6}$ is omniscient in $\mathcal{G}_{12}$.

- $\mathrm{T}_{4}^{(12)}$ : Indeed $\mathrm{w}_{12}=\mathrm{s}_{2}$ as shown above.

Therefore, if destination-to-source feedback is not available, the capacity region of this network is the region $\mathfrak{T}_{12}$ [7], and $\left(R_{1}, R_{2}\right)=(1,1 / 2)$ cannot be achieved. With feedback, however, this rate pair is achievable. Below we describe a simple scheme over two time slots to achieve it. See Fig. 3 for an illustration. We aim to deliver two binary symbols $\left\{a_{1}, a_{2}\right\}$ from $s_{1}$ to $d_{1}$ and one binary symbol $\{b\}$ from $s_{2}$ to $d_{2}$ over two time slots. At the first time slot, each node transmits what it receives except the node $\mathrm{u}_{2}$, with $\mathrm{s}_{1}$ transmitting $a_{1}$ and $\mathrm{s}_{2}$ transmitting $b$. The node $\mathrm{u}_{2}$ keeps silent at the first time slot. Hence $\mathrm{d}_{1}$ receives $a_{1}$, while $\mathrm{d}_{2}$ receives $a_{1} \oplus b$. At the second time slot, using the feedback from $d_{2}$, the source $s_{2}$ obtains $a_{1}$. At this time slot, each node transmits what it receives except the node $\mathrm{u}_{6}$, with $\mathrm{s}_{1}$ transmitting $a_{2}$ and $\mathrm{s}_{2}$ transmitting $a_{1}$. The node $u_{6}$ keeps silent at the second time slot. Hence $\mathrm{d}_{1}$ receives $a_{1} \oplus a_{2}$, while $\mathrm{d}_{2}$ receives $a_{1}$. Therefore, both user's symbols are delivered to their respective destinations successfully, and the rate pair $\left(R_{1}, R_{2}\right)=(1,1 / 2)$ is achieved.

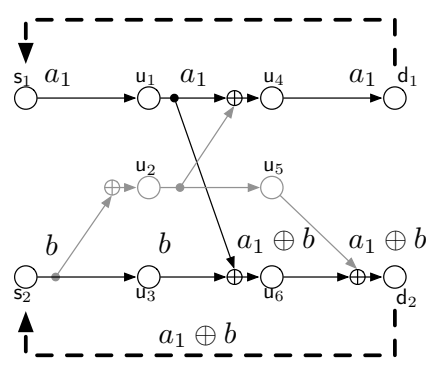

(a) Time Slot 1

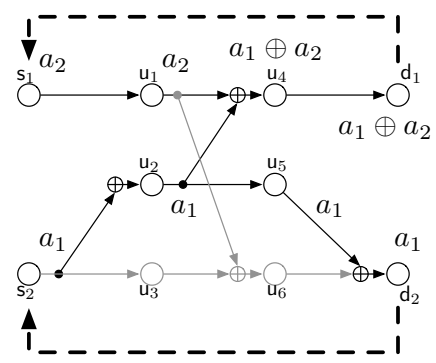

(b) Time Slot 2
Fig. 3. A Simple Scheme Achieving $(1,1 / 2)$

It remains to show that for any achievable $\left(R_{1}, R_{2}\right), 2 R_{1}+$ $2 R_{2} \leq 3$. We use the notation $A \stackrel{\mathrm{f}}{=} B$ to denote " $A$ is a function of $B$ ". Let us observe several functional relations. First, for the received signal at $\mathrm{d}_{1}$, we have $Y_{\mathrm{d}_{1}}^{N} \stackrel{\mathrm{f}}{=} Y_{\mathrm{u}_{4}}^{N}$, and for the received signal at $\mathrm{d}_{2}, Y_{\mathrm{d}_{2}}^{N} \stackrel{\mathrm{f}}{=}\left(Y_{\mathrm{u}_{6}}^{N}, X_{\mathrm{u}_{2}}^{N}\right)$. Second,

$$
\begin{aligned}
Y_{\mathrm{d}_{1}}^{N} & \stackrel{f}{=}\left(X_{\mathrm{u}_{1}}^{N}, X_{\mathrm{u}_{2}}^{N}\right) \stackrel{f}{=}\left(X_{\mathrm{u}_{3}}^{N}, Y_{\mathrm{u}_{6}}^{N}, X_{\mathrm{u}_{2}}^{N}\right) \stackrel{f}{=}\left(X_{\mathrm{s}_{2}}^{N}, Y_{\mathrm{u}_{6}}^{N}, X_{\mathrm{u}_{2}}^{N}\right) \\
& \stackrel{\mathrm{f}}{=}\left(W_{2}, Y_{\mathrm{d}_{2}}^{(N-1)}, Y_{\mathrm{u}_{6}}^{N}, X_{\mathrm{u}_{2}}^{N}\right) \stackrel{f}{=}\left(W_{2}, Y_{\mathrm{u}_{6}}^{N}, X_{\mathrm{u}_{2}}^{N}\right),
\end{aligned}
$$

where the key is that $X_{\mathrm{u}_{1}}^{N} \stackrel{\mathrm{f}}{=}\left(X_{\mathrm{u}_{3}}^{N}, Y_{\mathrm{u}_{6}}^{N}\right)$ due to the definition of channels. Third, since $Y_{\mathrm{d}_{i}}^{N} \stackrel{\mathrm{f}}{=}\left(X_{\mathrm{s}_{2}}^{N}, X_{\mathrm{s}_{1}}^{N}\right)$ for $i=1,2$,

$$
\begin{aligned}
Y_{\mathrm{d}_{2}}^{N} & \stackrel{f}{=}\left(X_{\mathrm{s}_{2}}^{N}, X_{\mathrm{s}_{1}}^{N}\right) \stackrel{\mathrm{f}}{=}\left(X_{\mathrm{s}_{2}}^{N}, W_{1}, Y_{\mathrm{d}_{1}}^{(N-1)}\right) \\
& \stackrel{\mathrm{f}}{=}\left(X_{\mathrm{s}_{2}}^{N}, W_{1}, X_{\mathrm{s}_{1}}^{(N-1)}\right)
\end{aligned}
$$

and hence inductively we have $Y_{\mathrm{d}_{2}}^{N} \stackrel{f}{=}\left(X_{\mathrm{s}_{2}}^{N}, W_{1}\right)$. Finally,

$$
\begin{aligned}
& X_{\mathrm{u}_{2}}^{N} \stackrel{\mathrm{f}}{=}\left(X_{\mathrm{u}_{1}}^{N}, Y_{\mathrm{u}_{4}}^{N}\right) \stackrel{\mathrm{f}}{=}\left(X_{\mathrm{s}_{1}}^{N}, Y_{\mathrm{u}_{4}}^{N}\right) \stackrel{f}{=}\left(W_{1}, Y_{\mathrm{d}_{1}}^{(N-1)}, Y_{\mathrm{u}_{4}}^{N}\right) \\
& \stackrel{f}{=}\left(W_{1}, Y_{u_{4}}^{N}\right) \text {. }
\end{aligned}
$$

From the last functional relation, $H\left(X_{\mathrm{u}_{2}}^{N} \mid W_{1}, Y_{\mathrm{u}_{4}}^{N}\right)=0$ and hence

$$
\begin{aligned}
& H\left(X_{\mathrm{u}_{2}}^{N} \mid W_{1}\right) \leq H\left(X_{\mathrm{u}_{2}}^{N}, Y_{\mathrm{u}_{4}}^{N} \mid W_{1}\right) \\
& =H\left(X_{\mathrm{u}_{2}}^{N} \mid W_{1}, Y_{\mathrm{u}_{4}}^{N}\right)+H\left(Y_{\mathrm{u}_{4}}^{N} \mid W_{1}\right)=H\left(Y_{\mathrm{u}_{4}}^{N} \mid W_{1}\right) .
\end{aligned}
$$

If $\left(R_{1}, R_{2}\right)$ is achievable, from data processing inequality and Fano's inequality, we have

$$
\begin{aligned}
& N\left(2 R_{1}+R_{2}-\epsilon_{1, N}\right) \\
& \leq I\left(W_{1} ; Y_{\mathrm{d}_{1}}^{N}\right)+I\left(W_{1} ; Y_{\mathrm{d}_{1}}^{N}\right)+I\left(W_{2} ; Y_{\mathrm{d}_{2}}^{N}\right) \\
& \stackrel{(\mathrm{a})}{\leq} I\left(W_{1} ; Y_{\mathrm{u}_{6}}^{N}, X_{\mathrm{u}_{2}}^{N}, W_{2}\right)+I\left(W_{1} ; Y_{\mathrm{u}_{4}}^{N}\right)+I\left(W_{2} ; Y_{\mathrm{u}_{6}}^{N}, X_{\mathrm{u}_{2}}^{N}\right) \\
& \stackrel{(\mathrm{b})}{=} I\left(W_{1} ; Y_{\mathrm{u}_{6}}^{N}, X_{\mathrm{u}_{2}}^{N} \mid W_{2}\right)+I\left(W_{1} ; Y_{\mathrm{u}_{4}}^{N}\right)+I\left(W_{2} ; Y_{\mathrm{u}_{6}}^{N}, X_{\mathrm{u}_{2}}^{N}\right) \\
& =H\left(Y_{\mathrm{u}_{6}}^{N}, X_{\mathrm{u}_{2}}^{N} \mid W_{2}\right)+H\left(Y_{\mathrm{u}_{4}}^{N}\right)-H\left(Y_{\mathrm{u}_{4}}^{N} \mid W_{1}\right) \\
& \quad+H\left(Y_{\mathrm{u}_{6}}^{N}, X_{\mathrm{u}_{2}}^{N}\right)-H\left(Y_{\mathrm{u}_{6}}^{N}, X_{\mathrm{u}_{2}}^{N} \mid W_{2}\right) \\
& \stackrel{(\mathrm{c})}{\leq} H\left(Y_{\mathrm{u}_{4}}^{N}\right)-H\left(X_{\mathrm{u}_{2}}^{N} \mid W_{1}\right)+H\left(X_{\mathrm{u}_{2}}^{N}\right)+H\left(Y_{\mathrm{u}_{6}}^{N} \mid X_{\mathrm{u}_{2}}^{N}\right) \\
& =H\left(Y_{\mathrm{u}_{4}}^{N}\right)+H\left(Y_{\mathrm{u}_{6}}^{N} \mid X_{\mathrm{u}_{2}}^{N}\right)+I\left(W_{1} ; X_{\mathrm{u}_{2}}^{N}\right) \\
& \stackrel{(\mathrm{d})}{\leq} 2 N+I\left(W_{1} ; X_{\mathrm{s}_{2}}^{N}\right),
\end{aligned}
$$

where $\epsilon_{1, N} \rightarrow 0$ as $N \rightarrow \infty$. (a) is due to the above functional relations. (b) is due to the fact that $W_{1}$ and $W_{2}$ are independent. (c) is due to cancellation of terms and the fact that $H\left(X_{\mathrm{u}_{2}}^{N} \mid W_{1}\right) \leq H\left(Y_{\mathrm{u}_{4}}^{N} \mid W_{1}\right)$. (d) is due to the fact that $X_{\mathrm{u}_{2}}^{N}$ is a function of $X_{\mathrm{s}_{2}}^{N}$.

On the other hand,

$$
\begin{aligned}
& N\left(R_{2}-\epsilon_{2, N}\right) \leq I\left(W_{2} ; Y_{\mathrm{d}_{2}}^{N}\right) \\
& \stackrel{(\text { a) }}{\leq} I\left(W_{2} ; X_{\mathrm{s}_{2}}^{N}, W_{1}\right)=I\left(W_{2} ; X_{\mathrm{s}_{2}}^{N} \mid W_{1}\right)=H\left(X_{\mathrm{s}_{2}}^{N} \mid W_{1}\right),
\end{aligned}
$$

where $\epsilon_{2, N} \rightarrow 0$ as $N \rightarrow \infty$. (a) is due to the above functional relations. Combining the above two, we have

$$
\begin{aligned}
& N\left(2 R_{1}+2 R_{2}-\epsilon_{N}\right) \leq 2 N+I\left(W_{1} ; X_{\mathrm{s}_{2}}^{N}\right)+H\left(X_{\mathrm{s}_{2}}^{N} \mid W_{1}\right) \\
& =2 N+H\left(X_{\mathrm{s}_{2}}^{N}\right) \leq 3 N,
\end{aligned}
$$


where $\epsilon_{N}=\epsilon_{1, N}+\epsilon_{2, N} \rightarrow 0$ as $N \rightarrow \infty$. Proof complete.

It turns out that both the above scheme and the converse proof can be generalized. The next section is devoted to the achievability proof of Theorem 4.1 .

\section{Achievability Proof}

As mentioned in Remark 4.1, for the achievability part we only need to show that, for a network that belongs to the class $\mathrm{T}^{(12)} \backslash \mathrm{O}$, when feedback from destinations to their respective sources is available, the rate pair $(1,1 / 2)$ is achievable.

Following the same line as the achievability proof in the non-feedback case [7], we without loss of generality assume that $k_{1}^{*} \leq k_{2}^{*}$ and distinguish into two different cases: $\left.{ }^{1} 1\right)$ $0<k_{1}^{*}=k_{2}^{*}=k^{*}$, and 2) $0<k_{1}^{*}<k_{2}^{*}$.

\section{A. Case: $0<k_{1}^{*}=k_{2}^{*}=k^{*}$}

In this case, we recall a lemma from [7] which captures the pattern of networks belonging to $\mathrm{T}^{(12)} \backslash \mathrm{O}$ when the two critical nodes are in the same layer. Let $\mathcal{P}_{1}:=\mathcal{P}\left(\mathrm{v}_{1}^{*}\right) \backslash \mathcal{P}\left(\mathrm{v}_{2}^{*}\right)$, $\mathcal{P}_{2}:=\mathcal{P}\left(\mathrm{v}_{2}^{*}\right) \backslash \mathcal{P}\left(\mathrm{v}_{1}^{*}\right), \mathcal{P}_{12}:=\mathcal{P}\left(\mathrm{v}_{1}^{*}\right) \cap \mathcal{P}\left(\mathrm{v}_{2}^{*}\right)$.

Lemma 6.1 ([7]): When $k_{1}^{*}=k_{2}^{*}=k^{*}$ and there is no omniscient node, we have the following equivalence relation ${ }^{2}$ :

$$
\begin{aligned}
& \mathrm{T}^{(12)} \backslash \mathrm{O} \Longleftrightarrow \\
& \left\{\begin{array}{l}
\mathcal{P}_{1} \text { is } \mathrm{s}_{1} \text {-only-reachable } \\
\mathrm{C}\left(\mathrm{s}_{1}, \mathrm{~s}_{2} ; \mathcal{P}_{2}\right)=1, \mathrm{u}_{21}:=\operatorname{Pmc}\left(\mathcal{P}_{2}\right) \neq \mathrm{s}_{i}, i=1,2 \\
\mathcal{P}_{12} \text { is } \mathrm{s}_{2} \text {-only-reachable } \\
\mathcal{K}^{\mathrm{s}_{1}}\left(\mathrm{u}_{21}\right) \text { forms }\left(\mathrm{s}_{1} ; \mathrm{d}_{1}\right) \text {-vertex-cut. }
\end{array}\right.
\end{aligned}
$$

Using this lemma, below we prove that $(1,1 / 2)$ can be achieved using linear schemes over extension field $\mathbb{F}_{2^{q}}$ (where $q$ is the effective block length), as defined in [7].

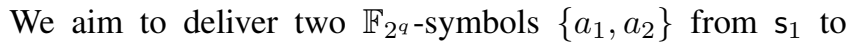
$\mathrm{v}_{1}^{*}$ and one symbol $b$ from $\mathrm{s}_{2}$ to $\mathrm{v}_{2}^{*}$ over two time slots, respectively. At time $t=1$, every node performs random linear coding (RLC, where each node transmits a uniformly randomly chosen linear transformation in $\mathbb{F}_{2^{q}}$ of its received symbol, as defined in [7]) with $\mathrm{s}_{1}$ sending $a_{1}$ and $\mathrm{s}_{2}$ sending $b$, except that the nodes in $\mathcal{P}_{12}$ have to keep silent. As a result, since $\mathcal{P}_{1}$ are $\mathrm{s}_{1}$-only-reachable and $\mathcal{P}_{12}$ are silent, $\mathrm{v}_{1}^{*}$ can decode $a_{1}$ with high probability and so can $\mathrm{d}_{1}$. $v_{2}^{*}$ (and hence $\mathrm{d}_{2}$ ), however, will receive a scaled version of the reception of $\mathrm{u}_{21}$. Since $\mathrm{u}_{21} \neq \mathrm{s}_{i}, i=1,2$, it is $\mathrm{s}_{1} \mathrm{~s}_{2}$-reachable, and hence $\mathrm{d}_{2}$ 's reception is a linear combination of $a_{1}$ and $b$. This linear combination is then fed back to $\mathrm{s}_{2}$, and it now knows $a_{1}$ before the transmission of time $t=2$.

At time $t=2$, every node performs RLC with $\mathrm{s}_{1}$ sending $a_{2}$ and $\mathrm{s}_{2}$ sending $a_{1}$, except that the nodes in $\mathcal{P}_{2}$ have to keep silent. Consequently, $v_{1}^{*}$ (and hence $d_{1}$ ) receives a linear combination of $a_{1}$ and $a_{2}$ and hence can decode $a_{2} \cdot v_{2}^{*}$ (and hence $\mathrm{d}_{2}$ ) receives a scaled version of $a_{1}$, and can decode $b$ by combining it with the previously received linear combination of $\left\{a_{1}, b\right\}$.

\footnotetext{
${ }^{1}$ Note that as pointed out in Lemma 3.1 in [7], if $k_{1}^{*}=0$ and there is no omniscient node, then $(1,1)$ is achievable.

${ }^{2}$ In [7], the left-hand side is originally written as $\mathrm{T}^{(12)}$ rather than $\mathrm{T}^{(12)} \backslash \mathrm{O}$, which is equivalent as under $k_{1}^{*}=k_{2}^{*}=k^{*}, \mathrm{~T}^{(12)} \cap \mathrm{O}=\emptyset$.
}

\section{B. Case: $0<k_{1}^{*}<k_{2}^{*}$}

Again we aim to deliver two symbols $\left\{a_{1}, a_{2}\right\}$ from $s_{1}$ to $\mathrm{d}_{1}$ and one symbol $b$ from $\mathrm{s}_{2}$ to $\mathrm{d}_{2}$ over two time slots. At time $t=1$, every node performs RLC with $\mathrm{s}_{1}$ sending $a_{1}$ and $\mathrm{s}_{2}$ sending $b$ except for one node, say $\mathrm{w}$, in $\mathcal{P}^{\mathrm{s}_{2}}\left(\mathrm{v}_{1}^{*}\right)$. This node $w \in \mathcal{P}^{\mathrm{s}_{2}}\left(\mathrm{v}_{1}^{*}\right)$ has to choose its scaling coefficient carefully so that the aggregate of $\mathcal{P}^{\mathrm{s}_{2}}\left(\mathrm{v}_{1}^{*}\right)$ 's transmission at $\mathrm{v}_{1}^{*}$ becomes 0 (interference neutralization). This is because under condition $\mathrm{T}^{(12)}, \mathcal{P}^{\mathrm{s}_{2}}\left(\mathrm{v}_{1}^{*}\right)$ are $\mathrm{s}_{2}$-only-reachable. Therefore, under the interference neutralization, in effect the network becomes $\mathcal{G}_{12}(\mathrm{w})$. In $\mathcal{G}_{12}(\mathrm{w})$ the destination $\mathrm{d}_{1}$ becomes $\mathrm{s}_{1}$ only-reachable. Hence $\mathrm{d}_{1}$ can decode $a_{1}$, while $\mathrm{v}_{2}^{*}$ receives a linear combination of $a_{1}$ and $b$. The coefficient of $b$ is non-zero with high probability since $v_{1}^{*}$ is not omniscient and therefore in $\mathcal{G}_{12}(\mathrm{w}), \mathrm{s}_{2}$ can reach $\mathrm{v}_{2}^{*}$ [7]. This linear combination is then fed back to $s_{2}$, and it now knows $a_{1}$ before the transmission of time $t=2$.

At time $t=2$, every node performs RLC with $\mathrm{s}_{1}$ sending $a_{2}$ and $\mathrm{s}_{2}$ sending $a_{1}$, except the nodes in $\mathcal{P}\left(\mathrm{v}_{2}^{*}\right)$. Since by the definition of critical nodes, $\mathrm{C}\left(\mathrm{s}_{1}, \mathrm{~s}_{2} ; \mathcal{P}\left(\mathrm{v}_{2}^{*}\right)\right)=2$, we can arrange their transmission carefully so that $\mathrm{v}_{2}^{*}$ can decode $a_{1}$. Hence, $v_{2}^{*}$ can decode $b$ by combining it with the previous received linear combination of $\left\{a_{1}, b\right\}$. Such arrangement in $\mathcal{P}\left(\mathrm{v}_{2}^{*}\right)$, as $\mathrm{v}_{2}^{*}$ is not omniscient, guarantees that $\mathrm{d}_{1}$ can still receive a linear combination of $a_{1}$ and $a_{2}$ with non-zero $a_{2-}$ coefficient [7]. Hence, $\mathrm{d}_{1}$ can decode $a_{2}$ as well.

\section{ACKNOWLEDGEMENT}

The author would like to thank Dr. Changho Suh for the fruitful discussion that sparked his interest in investigating the role of feedback in two unicast wireless networks. The work of I-Hsiang Wang was supported by EU project CONECT FP7-ICT-2009-257616.

\section{REFERENCES}

[1] C. Suh and D. N. C. Tse, "Feedback capacity of the Gaussian interference channel to within 2 bits," IEEE Transactions on Information Theory, vol. 57, pp. 2667-2685, May 2011.

[2] R. Etkin, D. N. C. Tse, and H. Wang, "Gaussian interference channel capacity to within one bit," IEEE Transactions on Information Theory, vol. 54, pp. 5534-5562, December 2008.

[3] A. S. Avestimehr, S. N. Diggavi, and D. N. C. Tse, "Wireless network information flow: A deterministic approach," IEEE Transactions on Information Theory, vol. 57, pp. 1872-1905, April 2011.

[4] G. Kramer, "Feedback strategies for white Gaussian interference networks," IEEE Transactions on Information Theory, vol. 48, pp. 1423 1438, June 2002.

[5] C. S. Vaze and M. K. Varanasi, "The degrees of freedom region of the MIMO interference channel with Shannon feedback," Arxiv Preprint, October 2011, http://arxiv.org/abs/1109.5779.

[6] S. Mohajer, R. Tandon, and H. V. Poor, "On the feedback capacity of the fully connected $K$-user interference channel," Arxiv Preprint, October 2011, http://arxiv.org/abs/1110.6487.

[7] I.-H. Wang, S. U. Kamath, and D. N. C. Tse, "Two unicast information flows over linear deterministic networks," Proceedings of IEEE International Symposium on Information Theory, pp. 2464-2468, August 2011. Extended version: http://arxiv.org/abs/1105.6326.

[8] I. Shomorony and A. S. Avestimehr, "Two-unicast wireless networks: Characterizing the degrees-of-freedom," Submitted to IEEE Transactions on Information Theory, March 2011, http://arxiv.org/abs/1102.2498.

[9] I.-H. Wang, "On two unicast wireless networks with destination-to-source feedback," Preprint, February 2012.

Link available at http://sites.google.com/site/ihsiangw/publication. 